\title{
Lagrangian study of surface transport in the Kuroshio Extension area based on simulation of propagation of Fukushima-derived radionuclides
}

\author{
S. V. Prants, M. V. Budyansky, and M. Yu. Uleysky \\ Laboratory of Nonlinear Dynamical Systems, Pacific Oceanological Institute of the Russian Academy of Sciences, \\ 43 Baltiyskaya st., 690041 Vladivostok, Russia \\ Correspondence to: S. V. Prants (prants@ poi.dvo.ru)
}

Received: 22 August 2013 - Revised: 24 December 2013 - Accepted: 14 January 2014 - Published: 24 February 2014

\begin{abstract}
Lagrangian approach is applied to study nearsurface large-scale transport in the Kuroshio Extension area using a simulation with synthetic particles advected by AVISO altimetric velocity field. A material line technique is proposed and applied to find out the origin of water masses in cold-core cyclonic rings pinched off from the jet in summer 2011. Tracking and Lagrangian maps provide the evidence of cross-jet transport. Fukushima-derived caesium isotopes are used as Lagrangian tracers to study transport and mixing in the area a few months after the 11 March 2011 tsunami that caused heavy damage of the Fukushima Nuclear Power Plant (FNPP). Tracking maps are computed to trace the origin of water parcels with measured levels of ${ }^{134} \mathrm{Cs}$ and ${ }^{137} \mathrm{Cs}$ concentrations collected during two research vessel (R/V) cruises in June and July 2011 in the large area of the northwest Pacific (Kaeriyama et al., 2013; Buesseler et al., 2012). It is shown that Lagrangian simulations are useful for finding the surface areas that are potentially dangerous due to the risk of radioactive contamination. The results of simulation are supported by tracks of the surface drifters that were deployed in the area.
\end{abstract}

\section{Introduction}

The Kuroshio Extension (KE) prolongs the Kuroshio Current, a western boundary current in the northwest Pacific, where the latter separates from the continental shelf of the Japanese island Honshu at Cape Inubō at about $35^{\circ} 42^{\prime} \mathrm{N}$. It flows eastward from this point as a strong unstable meandering jet constituting a front separating the warm subtropical and cold subpolar waters of the North Pacific Ocean. There are cyclonic and anticyclonic recirculation gyres on the northern and southern flanks of the jet. The main features of the KE are described by Qiu and Chen (2005) and Itoh and Yasuda (2010). The Kuroshio and the KE transport a large amount of heat and release that to the atmosphere, strongly affecting climate. It is a region with one of the most intense air-sea heat exchanges and the highest eddy kinetic energy level. It is also a region with commercial fishing grounds of Pacific saury, tuna, squid, Japanese sardine and other species.

The Kuroshio-Oyashio frontal zone contains various types of mesoscale and submesoscale eddies that transfer heat, salt, nutrients, carbon, pollutants and other tracers across the ocean. They originate, besides from the KE, from the Tsugaru Warm Current, flowing between the Honshu and Hokkaido islands, and from the cold Oyashio Current flowing out of the Arctic along the Kamchatka Peninsula and the Kuril Islands. Those eddies may persist for the periods ranging from a few weeks to a couple of years and have a strong influence on the local climate, hydrography and fishery.

A study of the role of KE rings and their interaction with the mean flow is important for many reasons. They act to transfer energy to the mean currents, influence on the KE jet dynamics and drive the recirculation gyres. They transport for a long distance water masses with biophysical properties different from ambient waters that may have a great impact on living organisms. The strongest mesoscale eddies of both polarities are generated along the KE. The warm-core anticyclonic rings (ACRs) are pinched off from the meandering KE mainly to the north whereas the cold-core cyclonic ones (CR) to the south of it. The occurrence, distribution and 
behavior of the KE rings, moving generally westward due to the planetary $\beta$ effect, have been studied in a number of papers via hydrographic observations, infrared imaging and altimetry data (Tomosada, 1986; Ebuchi and Hanawa, 2001; Waseda, 2003; Itoh and Yasuda, 2010). However, the process of their separation from the parent jet is not fully understood.

Lagrangian tools have been successfully used to obtain a detailed description of different advective transport phenomena in the ocean and atmosphere. There is a vast literature on this topic (for a review see Mancho et al., 2004; Wiggins, 2005; Koshel' and Prants, 2006; Prants, 2013, and references therein). As for the problem of eddy separation from strong jet currents and a cross-jet transport, there are papers on Lagrangian approach to the loop current eddy separation in the Gulf of Mexico (Kuznetsov et al., 2002; AndradeCanto et al., 2013) and on Lagrangian description of crossjet transport in the Kuroshio Current (Mendoza et al., 2010; Mendoza and Mancho, 2012). Near-surface velocity fields from numerical models of circulation in the Gulf of Mexico have been used to study the eddy separation process by computing effective invariant manifolds (Kuznetsov et al., 2002) and finite-time Lyapunov exponents (Andrade-Canto et al., 2013). It has been shown that the Lagrangian methods are a useful supplement to traditional approaches as they reveal flow details not easily extracted from Eulerian point of view.

In Mendoza et al. (2010) and Mendoza and Mancho (2012), a special lobe technique from dynamical system theory (Wiggins, 1992) and a method of distinguished hyperbolic trajectories (Ide et al., 2002) have been applied to find a geometrical skeleton of some transport processes in an altimetric velocity field in the Kuroshio region including surface cross-jet transport. In Samelson and Wiggins (2006), Uleysky et al. (2007), Uleysky et al. (2010a) and Uleysky et al. (2010b), a dynamical system-theory-based analysis, revealing mechanisms of chaotic zonal and cross-jet transport, has been carried out for a few kinematic and dynamical analytic models of meandering jets.

In this paper we study numerically the process of interaction of cold-core CRs with the KE main current, the events of their separation from the parent jet and their role in near-surface cross-jet transport. The special aim is to know whether it was possible for Fukushima-derived radionuclides to cross the KE jet, which is supposed to be an impenetrable barrier. Simulation is based on solving advection equations for synthetic particles in the AVISO velocity fields. The results are plotted as (1) backward-in-time Lagrangian latitudinal maps, where colors code the latitudes from which particles in a given region came to their final positions on the map, and (2) tracking maps showing where they were walking and how frequently they visited different places in the region for a given period of time. In Sect. 3.1 we compute both types of the maps to trace origin of water masses inside two CRs pinched off from the KE jet in summer 2011 and to document the surface cross-jet transport. The Fukushima-derived Cs isotopes are used as Lagrangian tracers to study transport and mixing processes. We apply the proposed material line technique in Sect. 3.2 to trace the origin of water parcels with measured levels of concentrations of Fukushima-derived Cs isotopes collected during two R/V cruises in June and July 2011 in the large area of the northwest Pacific (Kaeriyama et al., 2013; Buesseler et al., 2012). The results of our simulation are supported by tracks of the surface drifters that were deployed in the area.

\section{Data and methodology}

Geostrophic velocities were obtained from the AVISO database (http://www.aviso.oceanobs.com). The data are gridded on a $1 / 3^{\circ} \times 1 / 3^{\circ}$ Mercator grid. It has been justified by Mancho et al. (2006) that a bicubical spatial interpolation and third-order Lagrangian polynomials in time are able to provide accurate numerical results. Geostrophic velocities, derived from altimetry, are a good approximation to the surface fields. We do not expect essentially different results with respect to the present analysis, taking into account ageostrophic corrections such as an Ekman correction from wind data, because we are interested in transport phenomena for a comparatively long period of time, more than a few weeks. In fact, we study transport of particles and Fukushima-derived radionuclides for 3 months or more. It is reasonably to expect that wind variations are averaged out for such a long period of time.

Lagrangian trajectories are computed by integrating the advection equations with a fourth-order Runge-Kutta scheme with a fixed time step of 0.001th part of a day. Our results are based not on individual trajectories but on statistics for thousands and millions of trajectories. Most of them have been found to be chaotic with positive values of the maximal finite-time Lyapunov exponent. We cannot, of course, guarantee that we compute "true" trajectories for individual particles. We can only guarantee with our tracking and other Lagrangian maps that particles have visited some areas more frequently than the other ones. Typical chaotic systems are highly robust against a high-frequency noise mimicking small-scale diffusion. Thus, an additional small noise in the advection equations is not expected to change our results based on statistics for a large number of particles.

Satellite altimetry demonstrates clearly that the KE alternates between two dominant states: one with two quasistationary meanders and another one when the meanders are not especially prominent (Qiu and Chen, 2005; Sugimoto and Hanawa, 2012). During the meandering state, steep troughs develop stimulating ring pinch-off events on both flanks of the jet. In this paper we will focus on that state with increased eddy activity. A sketch of the meandering KE state in Fig. 1 shows the eastward jet current with two crests near $x=143^{\circ} \mathrm{E}$ and $x=151^{\circ} \mathrm{E}$, which are anticyclonic side of the jet and two troughs near $x=147^{\circ} \mathrm{E}$ and $x=153^{\circ} \mathrm{E}$ with cyclonic rotations. In reality the KE jet is highly unstable; the 


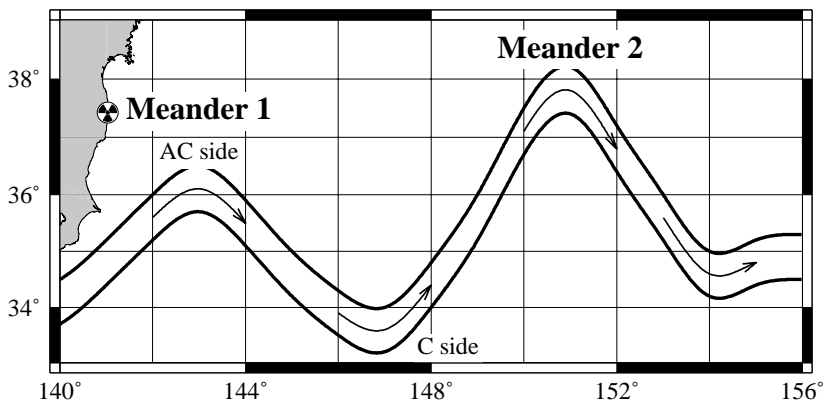

Fig. 1. Schematic view of the Kuroshio Extension state with two quasistationary meanders. Location of the Fukushima Nuclear Power Plant is shown.

meander's amplitude may change in the course of time, and locations of the crests and troughs may fluctuate strongly both in meridional and zonal directions.

Motion of a fluid particle in a two-dimensional flow is the trajectory of a dynamical system with given initial conditions governed by the velocity field. We solve advection equations on the Earth sphere in the form

$\dot{x}=u(x, y, t), \quad \dot{y}=v(x, y, t)$,

where time $t$ is in days. Coordinates $x$ and $y$ of a passive particle are related with its latitude $\phi$ and longitude $\lambda$ in degrees as follows:

$\lambda=\frac{x}{60}, \quad \phi=\frac{180}{\pi} \arcsin \tanh \left(\frac{\pi}{180}\left(\frac{y}{60}+y_{0}\right)\right)$,

$y_{0}=\frac{180}{\pi} \operatorname{artanh} \sin \left(\frac{\pi}{180} \phi_{0}\right), \quad \phi_{0}=-82$.

We use the transformation (Eq. 2) because the AVISO grid is homogeneous in those coordinates. The velocities $u$ and $v$ in Eq. (1) are expressed through the the latitudinal $U_{\phi}$ and longitudinal $U_{\lambda}$ components of the linear velocity $U$ in $\mathrm{cm} \mathrm{s}^{-1}$ as follows:

$u=\frac{10800}{\pi R_{\mathrm{E}} \cos \phi} \frac{86400}{100000} U_{\lambda} \approx \frac{0.466}{\cos \phi} U_{\lambda}$,
$v=\frac{10800}{\pi R_{\mathrm{E}} \cos \phi} \frac{86400}{100000} U_{\phi} \approx \frac{0.466}{\cos \phi} U_{\phi}$,

where $R_{\mathrm{E}}$ is the Earth radius in $\mathrm{km}$.

The Lagrangian technique we use is based on calculation of particle motion in an altimetric velocity field forward and backward in time. When integrating advection equations (Eq. 1) forward in time, we compute particle trajectories to know their fate; when integrating them backward in time, we know origin of particles and their history. A graphic view of transport and mixing is provided by Lagrangian synoptic maps that are plots of one of the Lagrangian indicators versus particle's initial positions (Prants et al., 2011a, 2013; Prants, 2013). The region under study is seeded by a large number of synthetic particles whose trajectories are computed forward or backward in time for a given period of time. There are a number of Lagrangian indicators (or descriptors in terminology of Mendoza and Mancho, 2012) that may be used to characterize transport and mixing processes in the ocean and atmosphere (for a recent review see Mancho et al., 2013). Among them are zonal and absolute displacements of water parcels, the number of their cyclonic and anticyclonic rotations, vorticity, their residence time in a given area, the number of times particles visit different places in the region and others. The so-called M-function, which measures the Euclidean arc length of the curves outlined by trajectories for a finite-time interval (Madrid and Mancho, 2009), can be also used to plot the corresponding Lagrangian maps. Sometimes it is useful to compute composite maps with two or more Lagrangian indicators plotted together.

To compute the Lagrangian maps, we apply the methodology elaborated in our recent papers and applied to study transport and mixing processes in different basins, from marine bays (Prants et al., 2013) and seas (Prants et al., 2011a) to the ocean scale (Prants et al., 2011b; Prants, 2013). In the first part of Sect. 3.1 we calculate for a large number of particles the latitudes, $\phi$, from which each particle came to its final position and plot Lagrangian latitudinal maps in order to document a cross-jet transport. Similar latitudinal maps have been used in another context by Abraham and Bowen (2002) and d'Ovidio et al. (2010).

The material line technique developed in this paper is a tool to trace origin, history and fate of water masses. A large number of particles (markers) are placed on a material line, crossing a feature under study, and evolve backward in time. Different kinds of outputs can be obtained with the help of that technique. In the context of this study, it is useful to get as an output tracking maps showing by density plots where the corresponding markers were walking for a given period of time. It has been done in the second part of Sect. 3.1 with markers placed on material lines crossing two Kuroshio CRs. This method is especially useful if one places material lines along the transects with in situ measurements. In Sect. 3.2 we carry out such simulations with material lines placed along the transects in the northwest Pacific where concentrations of radioactive Cs isotopes have been measured in surface seawater during two R/V cruises in 2011 after the Fukushima Nuclear Power Plant (FNPP) accident. Our simulations are complemented by tracks of available surface drifters (http://www.aoml.noaa.gov/phod/dac/index.php).

\section{Results}

\subsection{Lagrangian study of origin of Kuroshio Extension rings and possibility of surface cross-jet transport}

Documenting separation of rings from jets, merging with jets and tracking their propagation are long-standing problems in oceanography. An altimeter velocity field along with drifter 

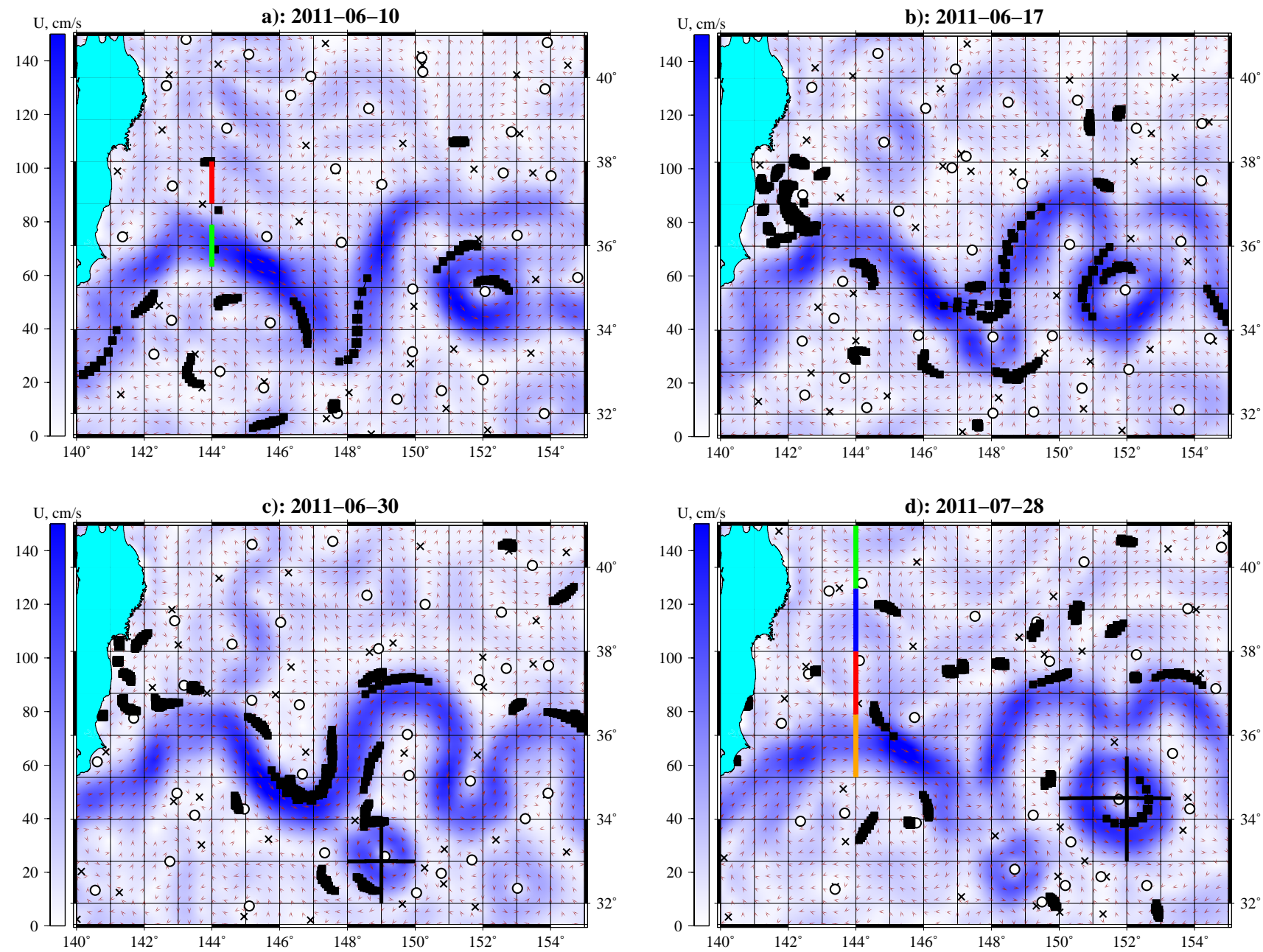

Fig. 2. (a)-(c) Metamorphoses of the velocity field in the process of formation of the CR1 on 10 June, 17 June and 30 June 2011 , respectively, with tracks of drifters imposed. (d) Velocity field on 28 July 2011 with the CR2 separated from the jet. Nuances of blue color measure the modulus of the linear velocity $U$ in $\mathrm{cm} \mathrm{s}^{-1}$. Crosses and open circles mark "instantaneous" hyperbolic and elliptic stagnation points, respectively. The straights are material lines that have been evolved backward in time to trace origin of the corresponding markers. Perpendicular black lines cross the CR1 and CR2. Colored lines in (a) and (d) are placed along the transects where seawater samples have been collected on 10 and 11 June 2011 during the R/V Ka'imikai-O-Kanaloa cruise (Buesseler et al., 2012) and on 26-29 July 2011 during the R/V Kaiun maru cruise (Kaeriyama et al., 2013), respectively.

observations may be used with these aims. Near-surface velocity fields on fixed days are shown in Fig. 2, where crosses and open circles mark "instantaneous" hyperbolic and elliptic stagnation points, respectively. Color codes the value of the modulus of the linear velocity $U=\sqrt{U_{\phi}^{2}+U_{\lambda}^{2}}$ in $\mathrm{cm} \mathrm{s}^{-1}$ Tracks of some surface drifters for 3 days are plotted by black squares.

In Fig. 2 we illustrate the process of formation of a $\mathrm{CR}$ (which we denote as CR1) from the KE meander in June 2011 when the KE was in the state with two prominent quasistationary meanders. In the beginning of June, the trough of the first meander starts to steepen with its edges becoming day by day closer and closer to each other (Fig. 2a). The edges merge eventually closing a volume of water with a cyclonic rotation to be connected with the parent jet by an arch (Fig. 2b). An elliptic point in its center and a hyperbolic point in the neck of the meander appear. Approximately 5 days later, the velocity field bifurcates, the CR1 is separated from the jet and the meander amplitude decreases correspondingly (Fig. 2c). Tracks of two drifters, encircling partly the ring, are shown in Fig. 2c.

There are a number of eddies around the KE. In this study we focus on the CR1, the CR2 (Fig. 2d), which is a ring separated from the jet on 28 July 2011, and the Tōhoku ACR, visible in all the panels of Fig. 2, with the center on 28 July 2011 to be at $144^{\circ} \mathrm{E}, 38^{\circ} \mathrm{N}$. Here we apply the material line technique to trace the origin and history of water masses inside the $\mathrm{CR} 1$ and $\mathrm{CR} 2$ crossing them by black perpendicular 
lines shown in Fig. 2c and d, respectively. In the next section we apply the same technique to trace the origin and history of markers placed on colored segments along the transects where seawater samples have been collected on 10 and 11 June 2011 during the R/V Ka'imikai-O-Kanaloa cruise (Buesseler et al., 2012) (Fig. 2a) and on 26-29 July 2011 during the R/V Kaiun maru cruise (Kaeriyama et al., 2013) (Fig. 2d).

Transport of water masses across strong jet currents, like the Gulf Stream and the Kuroshio, is important because they separate waters with distinct bio-physico-chemical properties. It may cause heating and freshening of waters with a great impact on the weather and living organisms. As for transport across the KE, one should mention the paper by Mendoza et al. (2010) in which a turnstile mechanism (Wiggins, 1992) has been supposed to provide KE crossjet transport in altimetric data sets. Mendoza et al. (2010) have computed stable and unstable manifolds of relevant distinguished hyperbolic trajectories and found a lobe transport across a Lagrangian barrier defined from pieces of those manifolds. It is a mechanism of chaotic advection well studied with analytical model flows (Wiggins, 1992; Koshel' and Prants, 2006). However, it is not direct evidence of crossjet transport because of difficulties and inevitable errors in computing the manifolds and relevant trajectories in altimetric data sets. Moreover, the cross-jet transport has been found by Mendoza et al. (2010) in a far downstream region between 155 and $165^{\circ} \mathrm{E}$ where the $\mathrm{KE}$ jet is highly unstable and may even bifurcate.

The question of whether the much more stable upstream $\mathrm{KE}$ jet between 141 and $153^{\circ} \mathrm{E}$ is an impenetrable barrier for cross-jet transport is still open. The new aspect of that problem arose suddenly after the Fukushima accident on 11 March 2011. As common opinion (Buesseler et al., 2012), it is difficult to expect observation of Fukushima-derived radionuclides on the southern side of the KE jet. Could contaminant waters from the Fukushima area cross the KE jet and appear on the southern side of the jet or not?

In order to document directly the cross-jet transport of Fukushima-derived radionuclides, we compute a special kind of Lagrangian maps by integrating the advection equations (Eq. 1) backward in time from a fixed day till the day of the accident for a large number of particles distributed in the study area. The latitude, $\phi$, from which each particle came to its final position is coded by color. The latitudinal map on 30 June 2011 in Fig. 3a demonstrates that "red" waters crossed the latitude $31^{\circ} \mathrm{N}$ from the south, "green" waters crossed the latitude of the FNPP $\left(141^{\circ} 05^{\prime} \mathrm{E}, 37^{\circ} 25^{\prime} \mathrm{N}\right)$ from the north, whereas nuances of the grey color code the particles that originated from the latitudes between 31 and $37^{\circ} \mathrm{N}$. Zoom in Fig. 3b shows the CR1 with "green" water in its core (originated from the latitudes $>37^{\circ} \mathrm{N}$ ) that may contain increased concentration of Fukushima-derived radionuclides.

The simulation results are confirmed by tracks of two surface drifters that were trapped by the CR1 (Fig. 3b). The

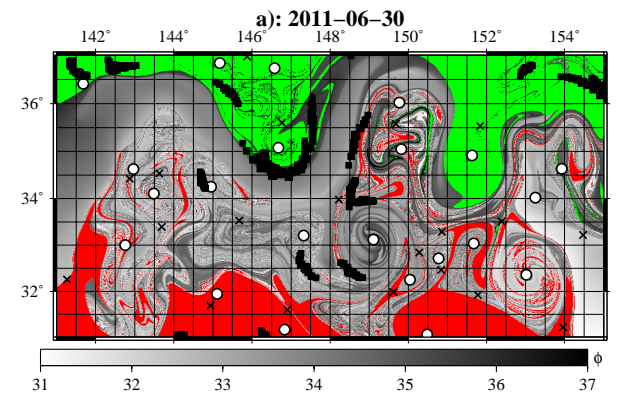

b)

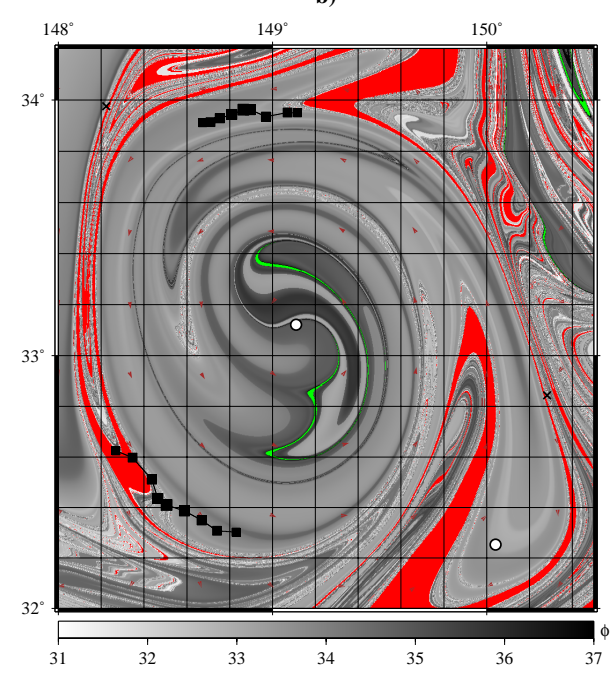

c)

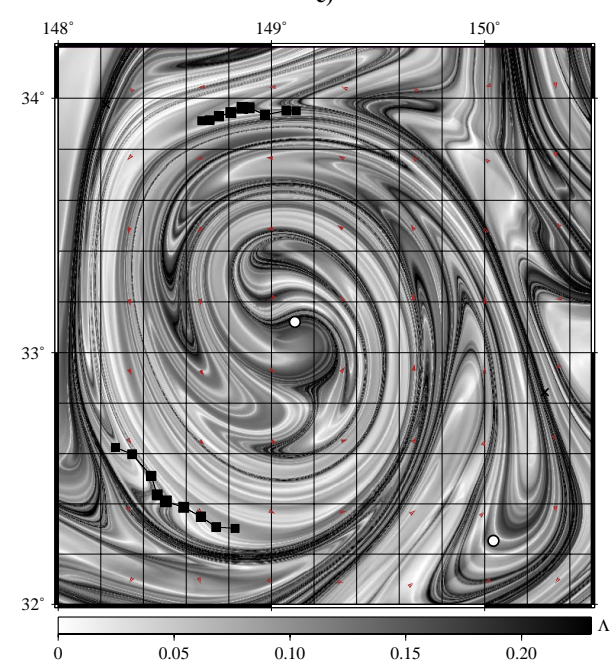

Fig. 3. (a) Lagrangian latitudinal map on 30 June 2011 computed from that date to the day of the accident, 11 March 2011, with "red" and "green" particles that originated from the latitudes $<31$ and $>37^{\circ} \mathrm{N}$, respectively. Nuances of the grey color in geographic degrees code the particles that originated from the latitudes between 31 and $37^{\circ} \mathrm{N}$. (b) Zoom of the area with the CR1 demonstrates transport across the KE jet ("green" particles). Tracks of two drifters to be trapped by the CR1 are shown as black squares. Crosses and open circles mark "instantaneous" hyperbolic and elliptic stagnation points, respectively. (c) Lyapunov map of the same area as in (b). 
southern track belongs to the drifter no. 98875 released on December 2010 at the point $126.784^{\circ} \mathrm{E}, 15.778^{\circ} \mathrm{N}$ (http: //www.aoml.noaa.gov/phod/dac/index.php). It was transported by the Kuroshio Current from the southwest. The northern track belongs to the drifter no. 36473 released on 11 June during the R/V Ka'imikai-O-Kanaloa cruise (Buesseler et al., 2012) at the point $144.087^{\circ} \mathrm{E}, 35.901^{\circ} \mathrm{N}$. It crossed the KE jet and was trapped by the CR1. It is a "green" drifter in Fig. 2 in Buesseler et al. (2012). Some of their "red" drifters in that Fig. 2 have been trapped by the meander trough that formed the CR2 in July 2011.

The map in Fig. $3 b$ is clear evidence of transport of water across the KE jet. To check that finding we initialized a material line crossing the $\mathrm{CR} 1$ core along $31^{\circ} 06^{\prime} \mathrm{N}$ (the horizontal black line in Fig. 2c) and evolved it backward in time till the day of the accident. It has been found that the fragments of that line, containing "green" particles, really came from the area near the location of the FNPP, whereas the other particles of that line came from the west, mainly along the KE jet. However, the amount of potentially dangerous Fukushima waters in the core of the CR1 is comparatively small.

To quantify chaos in irregular flows, it is useful to compute the maximal Lyapunov exponent that characterizes quantitatively mixing along with directions of maximal stretching and contracting (see, e.g., Koshel' and Prants, 2006). We show in Fig. 3c the map of the finite-time Lyapunov exponent, $\Lambda$, which is the finite-time average of the maximal separation rate for a pair of neighboring advected particles. That quantity has been computed backward in time from 30 June to 30 May by the method of the singular-value decomposition of an evolution matrix for the linearized advection equations (Prants et al., 2011a). The Lyapunov map demonstrates the same large-scale structures as the latitude map. It provides also a typical fine-scale pattern of mixing in the eddy CR1 with alternating black and white bands. The black bands are locus of those particles that separated from each other for 1 month in the past at a maximal rate. The boundaries between particles of different origin in Fig. $3 \mathrm{~b}$ correspond to the black bands with high values of $\Lambda$ in Fig. 3c.

Now we apply the material line technique to trace the origin of water masses in the CR1 and the CR2 that was born after a separation of the trough of the second KE meander from the parent jet in July 2011. In June-July 2011 it was deforming strongly and eventually produced a ring-like structure with a diameter of about $300 \mathrm{~km}$ that has been detached to the south of the parent jet and then reabsorbed in a short time. That ring-like structure with the center near $x=152^{\circ} \mathrm{E}$ and $y=34^{\circ} 30^{\prime} \mathrm{N}$ is seen in Fig. $2 \mathrm{~d}$ in the altimetric velocity field on 28 July 2011.

Starting on 30 June, we evolve backward in time until the day of the accident two perpendicular black material lines with a large number of markers, crossing the CR1 (Fig. 2c). The other two black material lines were chosen to cross the CR2 on 28 July (Fig. 2d), when a hyperbolic point appeared between the ring and the KE jet. We checked that the results did not change essentially when integrating not crossed segments but all the ring's interiors except for a larger density of points in some areas on the corresponding tracking maps.

Coming back to the question of whether the KE jet is an impenetrable barrier for Fukushima-derived radionuclides, we compute tracking maps. The region under study, 130$170^{\circ} \mathrm{E}$ and $25-50^{\circ} \mathrm{N}$, is divided into $500 \times 500$ cells. We advect particles of the corresponding material lines backward in time from 30 June (CR1) and from 28 July (CR2) until the day of the accident, 11 March 2011. Then one fixes each day how many times markers visited each cell during the month after the accident, from 11 March to 10 April, when the maximal leakage of radionuclides from the FNPP directly into the ocean and their atmospheric fallout on the ocean surface were registered. Summarizing those numbers, we get a total number of visits in each cell to be proportional to the density of marker's traces, $v$, which is coded by color in the logarithmic scale. The probability that the CR1 contains contaminated water is comparatively low because the density of points in the region around the FNPP is small (Fig. 4a). This map confirms the result of direct calculation of the cross-jet transport in Fig. 3b, where only a small amount of potentially contaminated "green" water, originated from the latitudes $>37^{\circ} \mathrm{N}$, is visible. It is clear from Fig. 4a that the CR1 consists mainly of Kuroshio water.

The method we proposed above provides "strict" proof of a cross-jet transport different from forward-in-time simulation of a patch of particles initially located around the Fukushima area. Previous modeling by Buesseler et al. (2012) (see Fig. S3 in a supplementary material of that paper) and Kaeriyama et al. (2013) (see their Fig. 2) has found some particles from the initial patch on the southern KE flank. However, day-by-day inspection of simulated propagation has shown that most of those particles, in fact, did not cross the jet. They propagated to the east along the northern KE side, and after reaching the longitude about $155^{\circ} \mathrm{E}$, where the KE becomes unstable and even may break down for a while, part of them were advected to the south and then to the west propagating along the southern KE flank. It is hardly to name "cross-jet transport". In order to document real cross-jet transport, it is necessary to select candidate particles on the southern KE flank and compute the corresponding longitudinal and tracking maps.

As to CR2 markers, the density of their traces in the area, which is supposed to be contaminated, is much higher as compared to the CR1 case (Fig. 4b). It means that the probability of observing higher concentrations of Fukushimaderived radionuclides in surface waters of the CR2 is expected to be comparatively high. The CR2 contains water parcels that moved during the month after the accident around the mesoscale eddies present to the north and east of the FNPP location. On the day of the accident, there was the eddy system with the large Tōhoku ACR with the center around $144^{\circ} \mathrm{E}$ and $39^{\circ} \mathrm{N}$, a small anticyclonic eddy to the north of it and a medium cyclonic eddy at the traverse of 

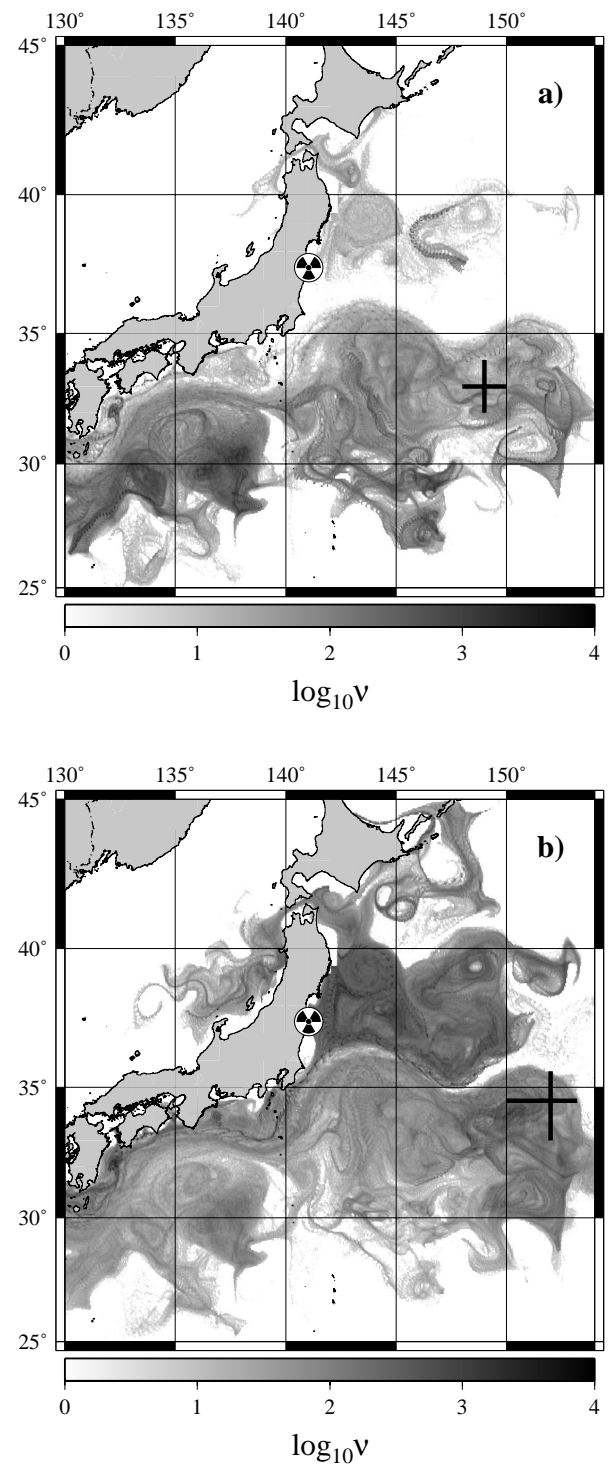

Fig. 4. Tracking maps for the markers placed on perpendicular material lines crossing (a) CR1 (Fig. 2c) and (b) CR2 (Fig. 2d). The maps show where the corresponding markers were walking after the Fukushima Nuclear Power Plant accident, from 11 March to 10 April 2011. The density of traces is in the logarithmic scale.

the Tsugaru Strait. It has been shown in our paper (Prants et al., 2011b) that namely this eddy system has governed mixing and transport of radioactive water, part of which has been trapped by the Tōhoku ACR and advected around the adjacent eddies to the north. The concentration of radionuclides around those eddies might be significantly greater than in other places because they are able to trap ambient contaminated waters (Prants et al., 2011b). The influence of the Tōhoku ACR is evident on both the tracking maps as a patch with increased density of traces at its place.

We conclude this section by emphasizing that the material line technique may be useful for finding the surface areas in the North Pacific that are potentially dangerous due to the risk of radioactive contamination. Before choosing the track of a planned $\mathrm{R} / \mathrm{V}$ cruise, it is instructive to make a simulation by initializing backward-in-time evolution of material lines, crossing eddies in the region visible on Lagrangian maps. The corresponding tracking maps computed would help to know where one could expect higher or lower concentrations of Fukushima-derived radionuclides in this or that eddy.

\subsection{Fukushima-derived radionuclides as Lagrangian tracers}

In this section we apply the material line technique to trace the origin of water parcels with measured levels of ${ }^{134} \mathrm{Cs}$ and ${ }^{137} \mathrm{Cs}$ concentrations collected during two R/V cruises in June and July 2011 (Kaeriyama et al., 2013; Buesseler et al., 2012). Starting from the dates of sampling, we evolve backward in time material lines placed along the transects, where stations with collected surface water samples were located. Results of direct observation of radioactive Cs in surface seawater collected from R/V Kaiun maru in a broad area in the western and central North Pacific in July and October 2011 and July 2012 have been reported by Kaeriyama et al. (2013). In this study, we focus on the results of measurements to be carried out at their stations C43-C55 from 26 to 29 July 2011 along the $144^{\circ} \mathrm{E}$ meridian from 35 to $41^{\circ} \mathrm{N}$. That transect is shown in Fig. 2d. The measured ${ }^{137} \mathrm{Cs}$ concentrations at the stations C43-C55 have been found to be in the range from the background level $1.9 \pm 0.4 \mathrm{mBq} \mathrm{kg}^{-1}$ (station C52) to $153 \pm 6.8 \mathrm{mBq} \mathrm{kg}^{-1}$ (station $\mathrm{C} 47$ ). The ${ }^{134} \mathrm{Cs} /{ }^{137} \mathrm{Cs}$ ratio was close to 1 . The level of the concentration of the caesium isotopes in the sea surface waters in the North Pacific before the accident did not exceed $2-3 \mathrm{mBq} \mathrm{kg}^{-1}$.

We placed a material line along that transect and divided it into four colored segments: (1) the orange segment, $35^{\circ}-$ $36^{\circ} 30^{\prime} \mathrm{N}$, crossing the first meander's crest with initialization on 26 July; (2) the red segment, $36^{\circ} 30^{\prime}-38^{\circ} \mathrm{N}$, crossing the southern part of the Tōhoku ACR with initialization on 27 July; (3) the blue segment, $38^{\circ}-39^{\circ} 30^{\prime} \mathrm{N}$, crossing its northern part with initialization on $28 \mathrm{July}$; and (4) the green segment, $39^{\circ} 30^{\prime}-41^{\circ} \mathrm{N}$ with initialization on 29 July.

The tracking maps in Fig. 5, colored as the corresponding segments, show where markers were walking from 11 March to 10 April 2011. Markers from the orange segment, as expected, were advected to their places at the end of July mainly by the Kuroshio Current from the southwest and did not cross the latitude of the segment's northern end, $36^{\circ} 30^{\prime} \mathrm{N}$ (Fig. 5a). The risk of their radioactive contamination is small. It is confirmed by the measured ${ }^{137} \mathrm{Cs}$ concentrations at the stations C52-C55 of that segment to be $2-5 \mathrm{mBq} \mathrm{kg}^{-1}$ (Kaeriyama et al., 2013), which is slightly higher than the background level. Markers from the red segment have been found walking mainly in the area to the north of the latitude $36^{\circ} 30^{\prime} \mathrm{N}$ (Fig. 5b). Part of that segment crossed the Tōhoku ACR existing in the area from the day of the accident to the 

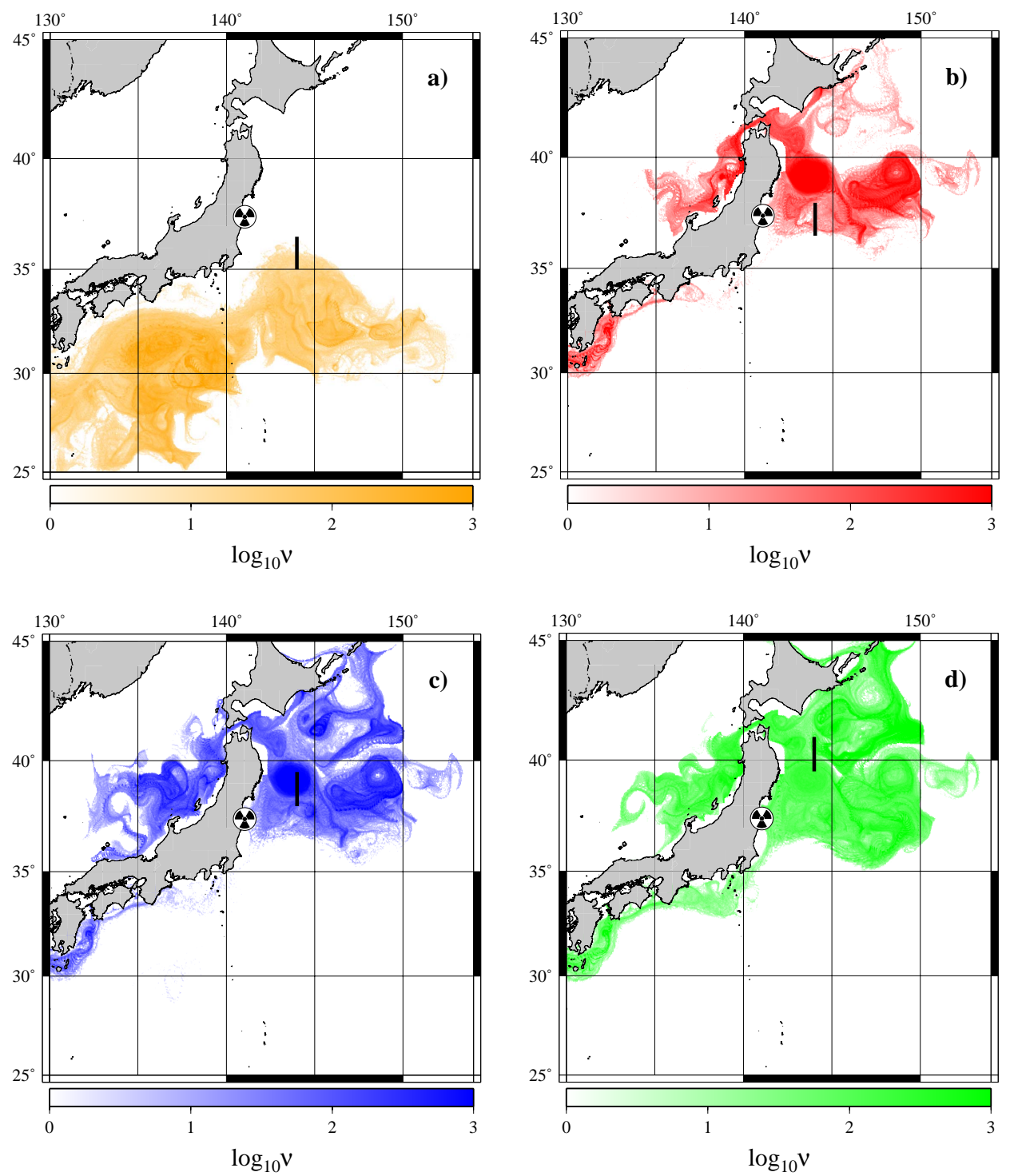

Fig. 5. Tracking maps for the markers placed on four material line segments along the transect where seawater samples have been collected on 26-29 July 2011 (Kaeriyama et al., 2013). The maps are colored as the corresponding segments in Fig. 2d and show where markers were walking from 11 March to 10 April 2011 . Markers were initially placed along the $144^{\circ} \mathrm{E}$ meridian at the following segments: (a) $35^{\circ}-$ $36^{\circ} 30^{\prime} \mathrm{N}$, (b) $36^{\circ} 30^{\prime}-38^{\circ} \mathrm{N}$, (c) $38^{\circ}-39^{\circ} 30^{\prime} \mathrm{N}$ and (d) $39^{\circ} 30^{\prime}-41^{\circ} \mathrm{N}$.

days of in situ measurements. That is why we see increased density of points in the area where it has been located in the range from 11 March to 10 April. A comparatively high level of Fukushima-derived caesium isotopes is expected in the corresponding water samples. It is really the case. The ${ }^{137} \mathrm{Cs}$ concentrations at the stations $\mathrm{C} 49$ and $\mathrm{C} 50$ of that segment were measured to be $36 \pm 3.3$ and $50 \pm 3.6 \mathrm{mBq} \mathrm{kg}^{-1}$ (Kaeriyama et al., 2013). The high caesium concentration levels up to $153 \pm 6.8 \mathrm{mBq} \mathrm{kg}^{-1}$ (Kaeriyama et al., 2013) have been measured at the stations $\mathrm{C} 46, \mathrm{C} 47$ and $\mathrm{C} 48$ (blue segment) and C43, C44 and C45 (green segment). The tracking maps in Fig. 5c and d show clearly an increased density of traces of the corresponding markers in the area where the maximal leakage of radionuclides from the FNPP directly into the ocean and their atmospheric fallout on the ocean surface were registered from 11 March to 10 April 2011. Those maps also demonstrate strong mixing in the KuroshioOyashio frontal zone. Traces of the markers, initialized at the end of July at a comparatively compact material line, have been found in the range 11 March-10 April in an extended area, including some parts of the Sea of Japan and the Okhotsk Sea.

Fukushima-derived ${ }^{134} \mathrm{Cs}$ and ${ }^{137} \mathrm{Cs}$ were measured in surface and subsurface waters, as well as in zooplankton and fish, at 50 stations on 4-18 June 2011 during the R/V Ka'imikai-O-Kanaloa cruise (Buesseler et al., 2012). We 
initialize a material line as shown in Fig. 2a where ${ }^{137} \mathrm{Cs}$ concentrations have been measured on 10 and 11 June at 25 stations in the range from $1.4 \pm 0.2 \mathrm{mBq} / \mathrm{kg}$ (station 13 ) to $173.6 \pm 9.9 \mathrm{mBq} \mathrm{kg}^{-1}$ (station 10). The ${ }^{134} \mathrm{Cs} /{ }^{137} \mathrm{Cs}$ ratio was close to 1 . Markers are placed on the green segment, $35^{\circ} 30^{\prime}-36^{\circ} 30^{\prime} \mathrm{N}$, crossing the first meander's crest (11 June), and the red one, $37-38^{\circ} \mathrm{N}$, to the north of the meander's crest (10 June). Traces of the markers of the green segment are found on both sides of the KE jet, whereas traces of the markers of the red one are on the northern side of the jet only. The southern part of the green segment crosses the jet itself, but its northern part is outside of it (Fig. 2a). That is why the tracking map in Fig. 6a consists of two disconnected domains: one is to the south of the jet and another one is to the north. The measured ${ }^{137} \mathrm{Cs}$ concentrations at the stations 13 and 14, situated in the green segment, were at the background level, in the range 1.4-3.6 $\mathrm{mBq} \mathrm{kg}^{-1}$ (Buesseler et al., 2012), because the corresponding markers were advected by the KE current (Fig. 6a).

Density of traces of the markers from the red segment is comparatively high in the area around the FNPP (Fig. 6b). This finding is confirmed by measurements at stations 10,11 and 12 (Buesseler et al., 2012) where the concentrations of Fukushima-derived ${ }^{134} \mathrm{Cs}$ and ${ }^{137} \mathrm{Cs}$ were in the range 21.9$173.6 \mathrm{mBq} \mathrm{kg}^{-1}$. The red segment crosses partly the Tōhoku ACR, which is visible in Fig. 2a, and the traces of its markers are dense at the place of that ring. Some markers were advected to their places on the initial segment by the Tsushima Current from the Sea of Japan to the Pacific Ocean through the Tsugaru Strait.

\section{Summary}

In this research, we used numerical simulations to study nearsurface large-scale transport in the KE area based on AVISO altimetric velocity field. After solving advection equations for passive particles backward in time, we have computed Lagrangian maps for their displacements and tracking maps for the number of times markers visited different places in the region.

Two KE cold-core rings, CR1 and CR2, were chosen to illustrate the process of pinching-off from the main jet in summer 2011. The tracking and Lagrangian maps were computed to trace the origin of water masses in the cores of those rings. They revealed near-surface cross-jet transport. This conclusion is supported by tracks of the surface drifters that were deployed in the area. Water masses, constituting the CR1, were advected mainly from the southwest by the Kuroshio and the KE, and only a small amount of its water was originated from the area to the north of the KE jet. Traces of the CR2 markers have been found in a large area to the south and the north of the jet, including the area around the FNPP location.
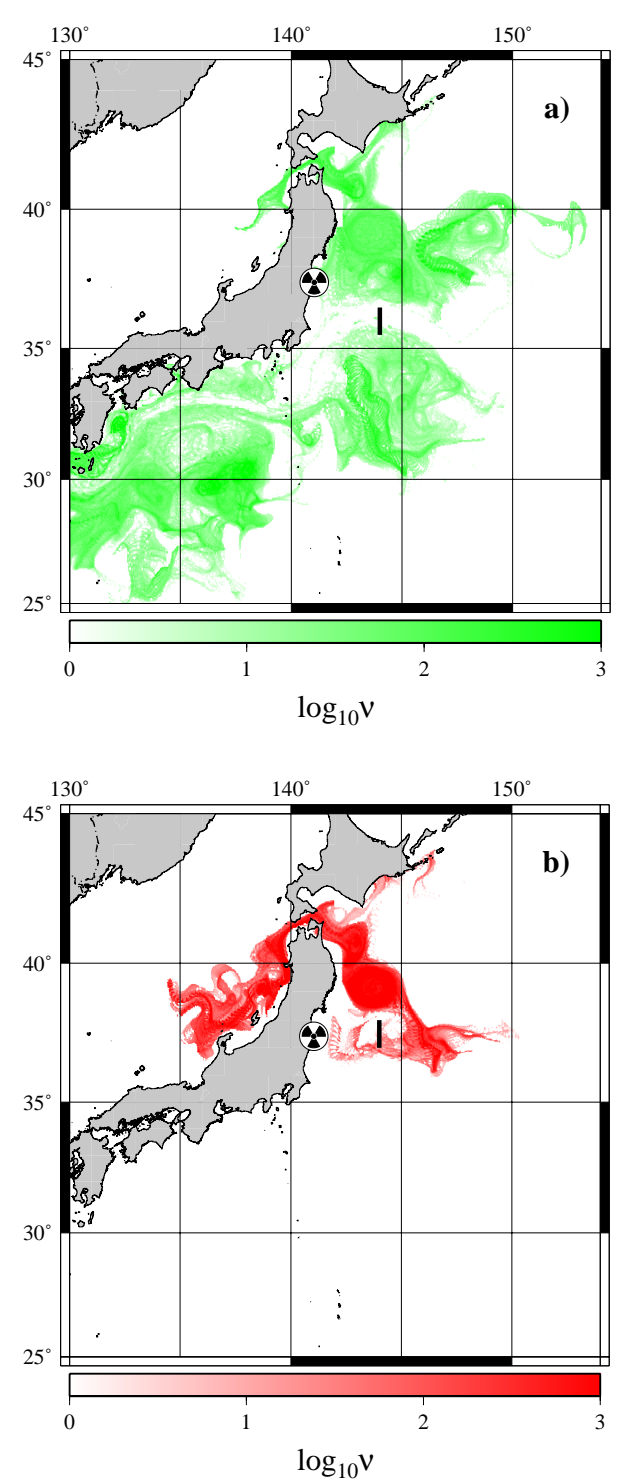

Fig. 6. Tracking maps for the markers placed on two material line segments along the transect where seawater samples have been collected on 10 and 11 June 2011 (Buesseler et al., 2012). The maps are colored as the corresponding segments in Fig. 2a and show where markers were walking from 11 March to 10 April 2011. Markers were initially placed along the $144^{\circ} \mathrm{E}$ meridian at the following segments: (a) $35^{\circ} 30^{\prime}-36^{\circ} 30^{\prime} \mathrm{N}$ and (b) $37^{\circ}-38^{\circ} \mathrm{N}$.

We used Fukushima-derived caesium isotopes as Lagrangian tracers comparing the results of our simulations with in situ observations of ${ }^{134} \mathrm{Cs}$ and ${ }^{137} \mathrm{Cs}$ concentrations in water samples collected during two R/V cruises in June and July 2011 (Kaeriyama et al., 2013; Buesseler et al., 2012). Evolving backward in time material lines along the transects where measurements have been carried out during those cruises, we computed the corresponding tracking maps. It is shown that the water parcels with caesium concentration, exceeding greatly the background level, were walking during 
1 month after the accident in the area where the leakage of radionuclides from the FNPP directly into the ocean and their atmosphere fallout on the ocean surface were registered to be maximal. The density of traces of markers with low caesium concentration in that area was found to be comparatively small.

We would like to emphasize that the tracking technique elaborated in this paper may be useful for planning $\mathrm{R} / \mathrm{V}$ cruises in the ocean. Before choosing the track of a planned $\mathrm{R} / \mathrm{V}$ cruise, it is instructive to make a simulation by initializing backward-in-time evolution of material lines, crossing potentially interesting coherent structures in the region visible on Lagrangian maps. The corresponding tracking maps would help to know where one could expect higher or lower concentrations of radionuclides, pollutants or other Lagrangian tracers.

In spite of inevitable and well-known errors in altimetryderived geostrophic velocity fields, the tracking technique seems to be the perspective in finding out the history and origin of water masses in coherent structures. The results obtained with that technique have been confirmed in this paper by a comparison with a large number of in situ measurements of concentration of Fukushima-derived radionuclides and drifter tracks. Measuring future concentrations of Fukushima-derived radionuclides and other pollutants and comparing those with the corresponding tracking maps, one gets an opportunity to estimate the temporal limits of the tracking technique.

Acknowledgements. This work was supported by the ICMAT Severo Ochoa project SEV-2011-0087 and the Russian Foundation for Basic Research (projects 11-01-12057, 12-05-00452, 13-0500099 and 13-01-12404). The altimeter products were distributed by AVISO with support from CNES.

Edited by: A. Turiel

Reviewed by: three anonymous referees

\section{References}

Abraham, E. R. and Bowen, M. M.: Chaotic stirring by a mesoscale surface-ocean flow, Chaos, 12, 373-381, doi:10.1063/1.1481615, 2002.

Andrade-Canto, F., Sheinbaum, J., and Zavala Sansón, L.: A Lagrangian approach to the Loop Current eddy separation, Nonlin. Processes Geophys., 20, 85-96, doi:10.5194/npg-20-85-2013, 2013.

Buesseler, K. O., Jayne, S. R., Fisher, N. S., Rypina, I. I., Baumann, H., Baumann, Z., Breier, C. F., Douglass, E. M., George, J., Macdonald, A. M., Miyamoto, H., Nishikawa, J., Pike, S. M., and Yoshida, S.: Fukushima-derived radionuclides in the ocean and biota off Japan, P. Natl. Acad. Sci., 109, 5984-5988, doi:10.1073/pnas.1120794109, 2012.
d'Ovidio, F., De Monte, S., Alvain, S., Dandonneau, Y., and Levy, M.: Fluid dynamical niches of phytoplankton types, P. Natl. Acad. Sci., 107, 18366-18370, doi:10.1073/pnas.1004620107, 2010.

Ebuchi, N. and Hanawa, K.: Trajectory of Mesoscale Eddies in the Kuroshio Recirculation Region, J. Oceanogr., 57, 471-480, doi:10.1023/A:1021293822277, 2001.

Ide, K., Small, D., and Wiggins, S.: Distinguished hyperbolic trajectories in time-dependent fluid flows: analytical and computational approach for velocity fields defined as data sets, Nonlin. Processes Geophys., 9, 237-263, doi:10.5194/npg-9-237-2002, 2002.

Itoh, S. and Yasuda, I.: Characteristics of Mesoscale Eddies in the Kuroshio-Oyashio Extension Region Detected from the Distribution of the Sea Surface Height Anomaly, J. Phys. Oceanogr., 40, 1018-1034, doi:10.1175/2009JPO4265.1, 2010.

Kaeriyama, H., Ambe, D., Shimizu, Y., Fujimoto, K., Ono, T., Yonezaki, S., Kato, Y., Matsunaga, H., Minami, H., Nakatsuka, S., and Watanabe, T.: Direct observation of ${ }^{134} \mathrm{Cs}$ and ${ }^{137} \mathrm{Cs}$ in surface seawater in the western and central North Pacific after the Fukushima Dai-ichi nuclear power plant accident, Biogeosciences, 10, 4287-4295, doi:10.5194/bg-10-4287-2013, 2013.

Koshel', K. V. and Prants, S. V.: Chaotic advection in the ocean, Physics-Uspekhi, 49, 1151-1178, doi:10.1070/PU2006v049n11ABEH006066, 2006.

Kuznetsov, L., Toner, M., Kirwan Jr., A., Jones, C., Kantha, L., and Choi, J.: The Loop Current and adjacent rings delineated by Lagrangian analysis of the near-surface flow, J. Mar. Res., 60, 405429, 2002.

Madrid, J. A. J. and Mancho, A. M.: Distinguished trajectories in time dependent vector fields, Chaos: An Interdisciplinary Journal of Nonlinear Science, 19, 013 111, doi:10.1063/1.3056050, 2009.

Mancho, A. M., Small, D., and Wiggins, S.: Computation of hyperbolic trajectories and their stable and unstable manifolds for oceanographic flows represented as data sets, Nonlin. Processes Geophys., 11, 17-33, doi:10.5194/npg-11-17-2004, 2004.

Mancho, A. M., Small, D., and Wiggins, S.: A comparison of methods for interpolating chaotic flows from discrete velocity data, Comput. Fluids, 35, 416-428, doi:10.1016/j.compfluid.2005.02.003, 2006.

Mancho, A. M., Wiggins, S., Curbelo, J., and Mendoza, C.: Lagrangian descriptors: A method for revealing phase space structures of general time dependent dynamical systems, Commun. Nonlin. Sci. Num. Simul., 18, 3530-3557, doi:10.1016/j.cnsns.2013.05.002, 2013.

Mendoza, C. and Mancho, A. M.: Review Article: "The Lagrangian description of aperiodic flows: a case study of the Kuroshio Current”, Nonlin. Processes Geophys., 19, 449-72, doi:10.5194/npg-19-449-2012, 2012.

Mendoza, C., Mancho, A. M., and Rio, M.-H.: The turnstile mechanism across the Kuroshio current: analysis of dynamics in altimeter velocity fields, Nonlin. Processes Geophys., 17, 103-111, doi:10.5194/npg-17-103-2010, 2010.

Prants, S. V.: Dynamical systems theory methods to study mixing and transport in the ocean, Physica Scripta, 87, 038115, doi:10.1088/0031-8949/87/03/038115, 2013. 
Prants, S. V., Budyansky, M. V., Ponomarev, V. I., and Uleysky, M. Yu.: Lagrangian study of transport and mixing in a mesoscale eddy street, Ocean Model., 38, 114-125, doi:10.1016/j.ocemod.2011.02.008, 2011a.

Prants, S. V., Uleysky, M. Y., and Budyansky, M. V.: Numerical simulation of propagation of radioactive pollution in the ocean from the Fukushima Dai-ichi nuclear power plant, Dokl. Earth Sci., 439, 1179-1182, doi:10.1134/S1028334X11080277, $2011 \mathrm{~b}$.

Prants, S. V., Ponomarev, V. I., Budyansky, M. V., Uleysky, M. Y., and Fayman, P. A.: Lagrangian analysis of mixing and transport of water masses in the marine bays, Izvest. Atmos. Ocean. Phys., 49, 82-96, doi:10.1134/S0001433813010088, 2013.

Qiu, B. and Chen, S.: Variability of the Kuroshio Extension Jet, Recirculation Gyre, and Mesoscale Eddies on Decadal Time Scales, J. Phys. Oceanogr., 35, 2090-2103, doi:10.1175/JPO2807.1, 2005.

Samelson, R. M. and Wiggins, S.: Lagrangian Transport in Geophysical Jets and Waves: The Dynamical Systems Approach (Interdisciplinary Applied Mathematics), Springer, New York, 2006.

Sugimoto, S. and Hanawa, K.: Relationship between the path of the Kuroshio in the south of Japan and the path of the Kuroshio Extension in the east, J. Oceanogr., 68, 219-225, doi:10.1007/s10872-011-0089-1, 2012.
Tomosada, A.: Generation and decay of Kuroshio warm-core rings, Deep-Sea Res. Pt. I, 33, 1475-1486, doi:10.1016/01980149(86)90063-4, 1986.

Uleysky, M. Y., Budyansky, M. V., and Prants, S. V.: Effect of dynamical traps on chaotic transport in a meandering jet flow, Chaos, 17, 043105, doi:10.1063/1.2783258, 2007.

Uleysky, M. Y., Budyansky, M. V., and Prants, S. V.: Chaotic transport across two-dimensional jet streams, J. Exp. Theor. Phys., 111, 1039-1049, doi:10.1134/S1063776110120174, 2010a.

Uleysky, M. Y., Budyansky, M. V., and Prants, S. V.: Mechanism of destruction of transport barriers in geophysical jets with Rossby waves, Phys. Rev. E, 81, 017202, doi:10.1103/PhysRevE.81.017202, 2010b.

Waseda, T., Mitsudera, H., Taguchi, B., and Yoshikawa, Y.: On the eddy-Kuroshio interaction: Meander formation process, J. Geophys. Res., 108, 3220, doi:10.1029/2002JC001583, 2003.

Wiggins, S.: Chaotic Transport in Dynamical Systems, in: Interdisciplinary applied mathematics, Springer-Verlag, New York, 1992.

Wiggins, S.: The dynamical systems approach to Lagrangian transport in oceanic flows, Annu. Rev. Fluid Mech., 37, 295-328, doi:10.1146/annurev.fluid.37.061903.175815, 2005. 